\title{
Scalar-tensor black holes coupled to Born-Infeld nonlinear electrodynamics
}

\author{
Ivan Zh. Stefanov*, Stoytcho S. Yazadjiev ${ }^{\dagger}$ \\ Dept. of Theoretical Physics, Faculty of Physics \\ St.Kliment Ohridski University of Sofia \\ 5, James Bourchier Blvd., 1164 Sofia, Bulgaria \\ Michail D. Todorov \\ Faculty of Applied Mathematics and Computer Science \\ Technical University of Sofia \\ 8, Kliment Ohridski Blvd., 1000 Sofia, Bulgaria
}

\begin{abstract}
The non-existence of asymptotically flat, neutral black holes and asymptotically flat, charged black holes in the Maxwell electrodynamics, with non-trivial scalar field has been proved for a large class of scalar-tensor theories. The noscalar-hair theorems, however, do not apply in the case of non-linear electrodynamics. In the present work numerical solutions describing charged black holes coupled to Born-Infeld type non-linear electrodynamics in scalar-tensor theories of gravity with massless scalar field are found. The causal structure and properties of the solutions are studied, and a comparison between these solutions and the corresponding solutions in the General Relativity is made. The presence of the scalar field leads to a much more simple causal structure. The present class of black holes has a single, non-degenerate horizon, i.e., its causal structure resembles that of the Schwarzschild black hole.
\end{abstract}

\section{Introduction}

Scalar-tensor theories of gravity are the most natural generalization of General Relativity (GR) and arise naturally in string theory and higher dimensional gravity theories [1]. Different modifications of scalar-tensor theories are attracting much interest also in cosmology and astrophysics. The most natural question is whether objects found and

\footnotetext{
*E-mail: zhivkov@phys.uni-sofia.bg

†E-mail: yazad@phys.uni-sofia.bg; Address since 1st April 200\%: Institut für Theoretische Physik, Universitat Gottingen, Friedrich-Hund-Platz 1, D-37077 Göttingen, Germany

${ }^{\ddagger}$ E-mail: mtod@tu-sofia.bg
} 
studied in the frame of GR would have different properties in the frame of the scalartensor theories. Such objects of interests are the black holes. A number of no-hair theorems concerning their global properties have been proved. The no-hair conjecture in GR says that in the exterior of a black hole the only information available regarding the black hole may be that of its mass, charge, and angular momentum. For neutral static, spherically symmetric black holes the only available information is about its mass, and the exterior of the black hole is reduced to the Schwarzschild solution. The natural question which arises then is whether the scalar field would lead to the existence of other preserved quantities which would allow a distant observer to distinguish between the Schwarzschild black hole and a black hole with a scalar dressing. Saa [2] was able to prove a no-scalar-hair theorem which rules out the existence of static, spherically symmetric, asymptotically flat, neutral black holes with regular, non-trivial scalar field for a large class of scalar tensor theories in which the scalar field is nonminimally coupled to gravity. He applied an explicit, covariant method to generate the exterior solutions for these theories through conformal transformations from the minimally coupled case. The scalar field in these theories becomes a constant and thus trivial if one demands that the essential singularity at the center of symmetry is hidden by an event horizon. A similar theorem treating also the case of charged scalar field with self-interaction was proved by Bekenstein [3]. Saa's theorem was generalized for the case of charged black holes in linear electrodynamics by Banerjee and Sen [4].

In the case of non-linear electrodynamics, however, the energy-momentum tensor of the electromagnetic field has a non-zero trace a sequence of which is that the electromagnetic field is non-trivially coupled to the scalar field. Hence, we can expect that the no-scalar-hair theorems might not hold in that case. In the present work we prove that our assumption is correct and find numerical solutions describing black holes with a non-trivial scalar field in the non-linear electrodynamics.

The nonlinear electrodynamics was first introduced by Born and Infeld in 1934 to obtain finite energy density model for the electron [5]. They proposed the following Lagrangian

$$
L_{B I}=2 b\left[1-\sqrt{1+\frac{1}{4 b} F_{\mu \nu} F^{\mu \nu}-\frac{1}{64 b^{2}}\left(F_{\mu \nu} \star F^{\mu \nu}\right)^{2}}\right],
$$

where the star " $\star$ " stands for the Hodge operator. In recent years nonlinear electrodynamics models are attracting much interest, too. The reason is that the nonlinear electrodynamics arises naturally in open strings and $D$-branes [6]. Nonlinear electrodynamics models coupled to gravity have been discussed in different aspects (see, for

example, [7]-[23] and references therein).

\section{Formulation of the problem}

The general form of the extended gravitational action in scalar-tensor theories is

$$
S=\frac{1}{16 \pi G_{*}} \int d^{4} x \sqrt{-\tilde{g}}\left(F(\Phi) \tilde{\mathcal{R}}-Z(\Phi) \tilde{g}^{\mu \nu} \partial_{\mu} \Phi \partial_{\nu} \Phi\right.
$$




$$
-2 U(\Phi))+S_{m}\left[\Psi_{m} ; \tilde{g}_{\mu \nu}\right]
$$

Here, $G_{*}$ is the bare gravitational constant, $\tilde{R}$ is the Ricci scalar curvature with respect to the space-time metric $\tilde{g}_{\mu \nu}$. The dynamics of the scalar field $\Phi$ depends on the functions $F(\Phi), Z(\Phi)$ and $U(\Phi)$. In order for the gravitons to carry positive energy the function $F(\Phi)$ must be positive. The nonnegativity of the energy of the dilaton field requires that $2 F(\Phi) Z(\Phi)+3[d F(\Phi) / d \Phi]^{2} \geq 0$. The action of matter depends on the material fields $\Psi_{m}$ and the space-time metric $\tilde{g}_{\mu \nu}$. It does not involve the scalar field $\Phi$ in order for the weak equivalence principle to be satisfied.

It is much more convenient from a mathematical point of view to analyze the scalar-tensor theories with respect to the conformally related Einstein frame given by the metric:

$$
g_{\mu \nu}=F(\Phi) \tilde{g}_{\mu \nu}
$$

Further, let us introduce the scalar field $\varphi$ (the so-called dilaton) via the equation

$$
\left(\frac{d \varphi}{d \Phi}\right)^{2}=\frac{3}{4}\left\{\frac{d \ln [F(\Phi)]}{d \Phi}\right\}^{2}+\frac{Z(\Phi)}{2 F(\Phi)}
$$

and define

$$
\mathcal{A}(\varphi)=F^{-1 / 2}(\Phi), 2 V(\varphi)=U(\Phi) F^{-2}(\Phi) .
$$

In the Einstein frame action (2) takes the form

$$
\begin{array}{r}
S=\frac{1}{16 \pi G_{*}} \int d^{4} x \sqrt{-g}\left[\mathcal{R}-2 g^{\mu \nu} \partial_{\mu} \varphi \partial_{\nu} \varphi-4 V(\varphi)\right] \\
+S_{m}\left[\Psi_{m} ; \mathcal{A}^{2}(\varphi) g_{\mu \nu}\right]
\end{array}
$$

where $R$ is the Ricci scalar curvature with respect to the Einstein metric $g_{\mu \nu}$. We take the following Jordan frame nonlinear electrodynamics action

$$
S_{m}=\frac{1}{4 \pi G_{*}} \int d^{4} x \sqrt{-\tilde{g}} L(X, Y)
$$

where

$$
X=\frac{1}{4} F_{\mu \nu} \tilde{g}^{\mu \alpha} \tilde{g}^{\nu \beta} F_{\alpha \beta}, \quad Y=\frac{1}{4} F_{\mu \nu}(\tilde{\star} F)^{\mu \nu}
$$

and " $\tilde{\star}$ " is the Hodge dual with respect to the Jordan frame metric $\tilde{g}_{\mu \nu}$.

In the Einstein frame we have

$$
S_{m}=\frac{1}{4 \pi G_{*}} \int d^{4} x \sqrt{-g} \mathcal{A}^{4}(\varphi) L(X, Y)
$$

where

$$
X=\frac{\mathcal{A}^{-4}(\varphi)}{4} F_{\mu \nu} g^{\mu \alpha} g^{\nu \beta} F_{\alpha \beta}, \quad Y=\frac{\mathcal{A}^{-4}(\varphi)}{4} F_{\mu \nu}(\star F)^{\mu \nu}
$$


and " $\star$ " stands for the Hodge dual with respect to the Einstein frame metric $g_{\mu \nu}$. The action (6) with (9) yields the following field equations

$$
\begin{aligned}
& \mathcal{R}_{\mu \nu}=2 \partial_{\mu} \varphi \partial_{\nu} \varphi+2 V(\varphi) g_{\mu \nu}-2 \partial_{X} L(X, Y)\left(F_{\mu \beta} F_{\nu}^{\beta}-\frac{1}{2} g_{\mu \nu} F_{\alpha \beta} F^{\alpha \beta}\right) \\
& -2 \mathcal{A}^{4}(\varphi)\left[L(X, Y)-Y \partial_{Y} L(X, Y)\right] g_{\mu \nu} \\
& \nabla_{\mu}\left[\partial_{X} L(X, Y) F^{\mu \nu}+\partial_{Y} L(X, Y)(\star F)^{\mu \nu}\right]=0 \\
& \nabla_{\mu} \nabla^{\mu} \varphi=\frac{d V(\varphi)}{d \varphi}-4 \alpha(\varphi) \mathcal{A}^{4}(\varphi)\left[L(X, Y)-X \partial_{X} L(X, Y)-Y \partial_{Y} L(X, Y)\right],
\end{aligned}
$$

where $\alpha(\varphi)=\frac{d \ln \mathcal{A}(\varphi)}{d \varphi}$.

In what follows we consider the truncated 1 Born-Infeld electrodynamics described by the Lagrangian

$$
L_{B I}(X)=2 b\left(1-\sqrt{1+\frac{X}{b}}\right) .
$$

Here $V(\varphi)$ will be equal to zero.

\section{Basic equations and qualitative investigation}

The metric of a static, spherically symmetric spacetime can be written in the form

$$
d s^{2}=g_{\mu \nu} d x^{\mu} d x^{\nu}=-f(r) e^{-2 \delta(r)} d t^{2}+\frac{d r^{2}}{f(r)}+r^{2}\left(d \theta^{2}+\sin ^{2} \theta d \phi^{2}\right)
$$

In the considered Born-Infeld type non-linear electrodynamics an electric-magnetic duality exists which means that the solutions in the magnetically charged case and the electrically charged case coincide. We will study the magnetically charged black holes for which the electromagnetic field is given by

$$
F=P \sin \theta d \theta \wedge d \phi
$$

and the magnetic charge is denoted by $P$.

The field equations reduce to the following coupled system of ordinary differential equations:

$$
\begin{aligned}
& \frac{d \delta}{d r}=-r\left(\frac{d \varphi}{d r}\right)^{2}, \\
& \frac{d m}{d r}=r^{2}\left[\frac{1}{2} f\left(\frac{d \varphi}{d r}\right)^{2}-\mathcal{A}(\varphi)^{4} L(X)\right], \\
& \frac{d}{d r}\left(r^{2} f \frac{d \varphi}{d r}\right)=r^{2}\left\{-4 \alpha(\varphi) \mathcal{A}^{4}(\varphi)\left[L(X)-X \partial_{X} L(X)\right]-r f\left(\frac{d \varphi}{d r}\right)^{3}\right\},
\end{aligned}
$$

\footnotetext{
${ }^{1}$ Here we consider the pure magnetic case for which $Y=0$.
} 
where $X$ reduces to:

$$
X=\frac{\mathcal{A}^{-4}(\varphi)}{2} \frac{P^{2}}{r^{4}} .
$$

In this paper, we will be searching for black hole solutions. We define black hole solutions as such solutions that have an event horizon on which the dilaton field $\varphi$ is regular. We will also require that $0<\mathcal{A}(\varphi)<\infty$ for $r_{H} \leq r \leq \infty$, where $r_{H}$ is the radius of the horizon. The latter condition ensures the regularity of the transition between the Einstein and the Jordan conformal frames. The diversity and the properties of the solutions depend strongly on the choice of the functions $\mathcal{A}(\varphi)$ (respectively on $\alpha(\varphi)$ ). In the present work we will consider only theories for which $\alpha(\varphi)$ has a fixed positive sign for all values of $\varphi$. The manner of investigation of solutions within theories with negative $\alpha(\varphi)$ is similar. Theories in which the coupling function changes its sign are much more complicated (also from numerical point of view) since in them some interesting effects like bifurcation of solutions can appear, especially when $\alpha(\varphi) \sim \varphi$. Such solutions are currently being studied by the authors and the results will be given elsewhere.

Some general properties of the solution can be derived through an analytical investigation of the equations. We will consider nonlinear electrodynamics for which the following relation holds

$$
X \partial_{X} L(X)-L(X)>0 .
$$

The Born-Infeld Lagrangian (12), belongs to the same class of nonlinear electrodynamics.

Using the following equation

$$
\frac{d}{d r}\left(e^{-\delta} r^{2} f \frac{d \varphi}{d r}\right)=4 r^{2} e^{-\delta} \alpha(\varphi) \mathcal{A}^{4}(\varphi)\left[X \partial_{X} L(X)-L(X)\right]>0
$$

which is another form of equation (17), we find that $e^{-\delta} r^{2} f \frac{d \varphi}{d r}$ increases monotonously and has one zero at most, if any. In case a black hole exists, $e^{-\delta} r^{2} f \frac{d \varphi}{d r}$ vanishes on the horizon. On the other hand, as we already noted, this expression is a monotonously increasing function of $r$. Therefore $e^{-\delta} r^{2} f \frac{d \varphi}{d r}<0$ and $e^{-\delta} r^{2} f \frac{d \varphi}{d r}>0$, inside and outside the horizon, respectively. In order for this to be true, $\frac{d \varphi}{d r}$ must be positive, which means that $\varphi$ increases monotonously.

The non-existence of inner horizons can be proved in another way. Let us admit that more than one horizon exists. Then, integrating equation (20) in the interval $r \in\left[r_{-}, r_{+}\right]$where we denote he first inner and the outer horizons with $r_{-}$and $r_{+}$, respectively, i.e.,

$$
\begin{array}{r}
\left.\left(e^{-\delta} r^{2} f \frac{d \varphi}{d r}\right)\right|_{r_{+}}-\left.\left(e^{-\delta} r^{2} f \frac{d \varphi}{d r}\right)\right|_{r_{-}} \\
=4 \int_{r_{-}}^{r_{+}} r^{2} e^{-\delta} \alpha(\varphi) \mathcal{A}^{4}(\varphi)\left[X \partial_{X} L(X)-L(X)\right] d r>0
\end{array}
$$


and having in mind that $f\left(r_{-}\right)=0=f\left(r_{+}\right)$, we reach a contradiction, which means that our admission is incorrect. So if a black hole exists it will have a single horizon, i.e., its causal structure will be Schwarzschild-like. In both conformal frames, inside the event horizon a space-like singularity is hidden.

The qualitative behavior of $\delta(r)$ can easily be seen from equation (15). It decreases monotonously with $r$.

\subsection{Numerical results}

The nonlinear system (15)-(17) is inextricably coupled and the event horizon $r_{H}$ is $a$ priori unknown boundary. In order to be solved, it is recast as a equivalent first order system of ordinary differential equations. Following the physical assumptions of the matter under consideration the asymptotic boundary conditions are set, i.e.,

$$
\begin{gathered}
\lim _{r \rightarrow \infty} m(r)=M \quad(M \text { is the mass of the black hole in the Einstein frame }), \\
\lim _{r \rightarrow \infty} \delta(r)=\lim _{r \rightarrow \infty} \varphi(r)=0 .
\end{gathered}
$$

At the horizon both the relationship

$$
f\left(r_{H}\right)=0
$$

and the regularization condition

$$
\left.\left(\frac{d f}{d r} \cdot \frac{d \varphi}{d r}\right)\right|_{r=r_{H}}=\left.\left\{4 \alpha(\varphi) \mathcal{A}^{4}(\varphi)\left[X \partial_{X} L(X)-L(X)\right]\right\}\right|_{r=r_{H}}
$$

concerning the spectral quantity $r_{H}$ must be held. For the treating the above posed boundary-value problem (BVP) the Continuous Analog of Newton Method (see, for example [24],[25],[18]) is used. After an appropriate linearization the original BVP is rendered to solving a vector two-point BVP. On a discrete level sparse (almost diagonal) linear algebraic systems with regard to increments of sought functions $\delta(r), m(r)$, and $\varphi(r)$ have to be inverted.

We studied the parametric space for fixed value of the coupling parameter $\alpha=0.01$ (this value is close to the one established on the bases of experimental data) and for several values of the magnetic charge. Constant coupling parameter corresponds to the Brans-Dicke theory. The behavior of the solutions for any $\alpha(\varphi)>0$ for which $\alpha(0)=0.01$ are qualitatively the same and quantitatively very close to the case we studied as the numerical investigations confirm.

Figures (1)-(4) show the value of the scalar field on the event horizon, the radius of the black hole in the Einstein and in the Jordan conformal frames, and the temperature of the horizon as a function of the mass of the black hole, respectively. For higher masses the behavior of the solutions resembles that of the GR case. When we decrease the mass for a fixed value of $P$, however, the solutions start to deviate from the GR case considerably. In GR [9] the properties of the solution depend on the value of following quantity

$$
M^{\prime}=M-\mathcal{E},
$$


where $M$ is the mass of the black hole and in our notations

$$
\mathcal{E}=\frac{P^{3 / 2}}{\sqrt{\frac{4 \pi}{\sqrt{2 b}}}} \frac{\pi^{3 / 2}}{3 \Gamma\left(\frac{3}{4}\right)^{2}} .
$$

For $M^{\prime} \geq 0$ a single non-degenerate horizon exists. The case $M^{\prime}<0$ resembles the Reissner-Nordström solution in which extremal black holes exist. In the case under consideration, however, the absolute value of the scalar field rises considerably and prevents the emergence of a degenerate horizon. This can be clearly seen from the behavior of the graph representing the case $P=3.5$ in Fig.(44). The temperature of the horizon approaches zero but then suddenly rises. For these values of the mass $M$ and the magnetic charge $P$ in GR an extremal solution is reached. Far from the extremal solution the dilaton becomes almost zero and remains constant with the increase of mass $M$.

It can also be noted in Fig.(2) that the radius of the horizon turns to zero for a finite value of the mass $M$ of the black hole. The mass-radius relation in the Jordan frame is shown in Fig.(3). For the values of the parameters we work with the difference between Figs. (2) and (3) is insignificant.

\section{Thermodynamics}

For the solution we study, in the Einstein frame, the First Law (FL) of thermodynamics holds. The formal derivation of this law can be seen in the work of Rasheed [26]. The presence of the scalar field leads to the existence of a new charge namely the dilaton charge which we define in the following way

$$
\mathcal{D}=-\left.r^{2} \frac{d \varphi}{d r}\right|_{r \rightarrow \infty} .
$$

This charge, however, is not independent and can be determined unambiguously once the mass and the magnetic charge of the black hole and the asymptotic value of the scalar field at infinity are known. The relation between the charges can be seen if we integrate equation (20) from the radius of the horizon to infinity. We obtain

$$
\mathcal{D}=4 \int_{r_{H}}^{\infty} r^{2} \alpha(\varphi) \mathcal{A}^{4}(\varphi)\left[X \partial_{X} L(X)-L(X)\right] d r .
$$

Since in the asymptotically flat case the scalar field is fixed and vanishing at infinity the term coming from the scalar field also vanishes and the FL of thermodynamics is the same as in the GR

$$
\delta M=T \delta S+\Psi_{H} \delta P,
$$

where $T, S$ and $P$ are the temperature, the entropy, and the magnetic charge of the black hole, respectively. The quantity $\Psi$ conjugate to the magnetic charge is the potential of the magnetic field which is given by the following definition

$$
H_{\mu}=\partial_{\mu} \Psi
$$


On the other hand the magnetic field is defined as

$$
H_{\mu}=-\star G_{\mu \nu} \xi^{\nu},
$$

where

$$
G_{\mu \nu}=-\frac{1}{2} \frac{\partial L}{\partial F_{\mu \nu}},
$$

$\xi=\frac{\partial}{\partial t}$ is the Killing vector generating time translations and " $\star$ " is the Hodge star operator.

Originally we formulated the theory in Jordan frame but studied the solutions in the conformally related Einstein frame not only for mathematical convenience but also because the FL of black hole thermodynamics is naturally connected with the Einstein frame as this can be seen below.

The temperature of the event horizon is invariant under conformal transformations of the metric that are unity at infinity [27. The properly defined entropy is also the same in both conformal frames. It has been proved that in the Jordan frame the entropy of the black hole is not simply one forth of the horizon area [28, 29] as in the Einstein frame and needs to be generalized. The entropy in the Jordan frame is defined as:

$$
S_{J}=\frac{1}{4 G_{*}} \int d^{2} x \sqrt{-^{(2)} \tilde{g}} F(\Phi) .
$$

Using relation (3) we find that

$$
S_{J}=\frac{1}{4 G_{*}} \int d^{2} x \sqrt{-^{(2)} g}=S_{E}=S .
$$

In the last two equations ${ }^{(2)} \tilde{g}$ and ${ }^{(2)} g$ are the determinants of the induced metrics on the horizon in the Jordan and in the Einstein frame, respectively.

The term in the FL (26) connected with the magnetic charge is also preserved under the conformal transformations.

In order for the FL of thermodynamics to be satisfied in the Jordan frame the mass should be properly chosen since the Arnowitt-Deser-Misner (ADM) masses in both frames are not equivalent. It can be easily shown that the ADM mass in the Jordan frame $M_{J}$ is related to both the ADM mass in the Einstein frame $M$ and the dilaton charge $\mathcal{D}$ in the following way

$$
M_{J}=M+\alpha \mathcal{D} .
$$

For the Jordan frame, the proper mass in the FL of thermodynamics is the ADM mass in the Einstein frame $M$. Similarly, for boson and fermion stars the proper measure for the energy of the system is again the ADM mass in the Einstein frame $M$. For more details on the subject we would refer the reader to the works [30, 31, 32, 33].

\section{Conclusion}

In the present work numerical solutions describing charged black holes coupled to non-linear electrodynamics in the scalar-tensor theories with massless scalar field were 
found. Since an electric-magnetic duality is present, in this work only purely magnetically case was studied. For the Lagrangian of the non-linear electrodynamics the truncated Born-Infeld Lagrangian was chosen and scalar-tensor theories with positive coupling parameter were considered. As a result of the numerical and analytical investigation, some general properties of the solutions were found. Due to the presence of the massless scalar field the found solutions have a single, non-degenerate event horizon, i.e., their causal structure resembles that of the Schwarzschild black hole and is simpler than the corresponding solution in GR. In both conformal frames, inside the event horizon a space-like singularity is hidden. Black hole thermodynamics was also discussed.

\section{Acknowledgments}

This work was partially supported by the Bulgarian National Science Fund under Grant MUF 04/05 (MU 408) and the Sofia University Research Fund N60. The final stage of this work was completed after S. Y. has started his visit in Institut für Theoretische Physik, Universitat Göttingen as an Alexander von Humboldt research fellow so this author would like to thank Alexander von Humboldt Foundation for the financial support and the Institut für Theoretische Physik for the hospitality.

\section{References}

[1] T. Damour and A. Polyakov, Nucl. Phys. B423, 532 (1994)

[2] A. Saa, J. Math. Phys. 37, 2346 (1996).

[3] A. Mayo and J. Bekenstein, Phys. Rev. D54, 5059 (1996).

[4] N. Banerjee and S. Sen, Phys. Rev. D58, 104024 (1998).

[5] M. Born and L. Infeld, Proc. R. Soc. (London) A144, 425 (1934).

[6] R. Leigh, Mod. Phys. Lett. A4, 2767 (1989).

[7] M. Demianski, Found.Phys. 16, 187 (1986)

[8] D. Wiltshire, Phys.Rev. D38, 2445 (1988)

[9] G. Gibbons and D. Rasheed, Nucl. Phys. B454, 185 (1995).

[10] G. Gibbons and D. Rasheed, Phys. Lett. B365, 46-50 (1996).

[11] E. Ayon-Beato, A. Garcia, Phys.Rev.Lett. 80, 5056 (1998)

[12] T. Tamaki, T. Torii, Phys.Rev. D62, 061501 (2000)

[13] T. Tamaki, T. Torii, Phys. Rev. D64, 024027 (2001) 
[14] G. Clement and D. Gal'tsov, Phys. Rev. D62, 124013 (2000).

[15] G. Gibbons and C. Herdeiro, Class. Quantum Grav. 18, 1677 (2001).

[16] H. Yajima and T. Tamaki, Phys. Rev. D63, 064007 (2001).

[17] R. Yamazaki and D. Ida, Phys. Rev. D64, 024009 (2001).

[18] S. Yazadjiev, P. Fiziev, T. Boyadjiev and M. Todorov, Mod. Phys. Lett. A16, 2143 (2001).

[19] M. Gurses and O. Sarioglu, Class. Quantum Grav. 20, 351 (2003).

[20] T. Dey, Phys. Lett. B595, 484 (2004).

[21] R. Cai, D. Pang and A. Wang, Rev. D70, 124034 (2004).

[22] S. Yazadjiev, Phys. Rev. D72, 044006 (2005)

[23] A. Sheykhi, N. Riazi and M. H. Mahzoon, Phys. Rev. D74, 044025 (2006)

[24] M. K. Gavurin, Izv. Vyssh. Uchebn. Zaved., Mat. 5(6), 18 (1958) (in Russian); (see also Mathematical Reviews 25, 1380 (1958)).

[25] E. P. Jidkov, G. I. Makarenko and I. V. Puzynin, in Physics of Elementary Particles and Atomic Nuclei (JINR, Dubna, 1973) vol. 4, part I, 127-166 (in Russian), English translation: American Institute of Physics, p.53.

[26] D. A. Rasheed, Non-Linear Electrodynamics: Zeroth and First Laws of Black Hole Mechanics, hep-th/9702087.

[27] T. Jacobsond and G. Kang, Class. Quant. Grav. 10 L201 (1993).

[28] M. Visser, Phys. Rev. D48, 583 (1993).

[29] L. H. Ford and Thomas A. Roman, Phys. Rev. D64, 024023 (2001).

[30] D. L. Lee, Phys. Rev. D 10, 2374 (1974).

[31] M. A. Scheel, S. L. Shapiro, and S. A. Teukolsky, Phys. Rev. D 51, 4208 (1995).

[32] A. W. Whinnett, Class. Quantum Grav. 162797 (1999).

[33] S. S. Yazadjiev, Class. Quantum Grav. 16 L63 (1999). 


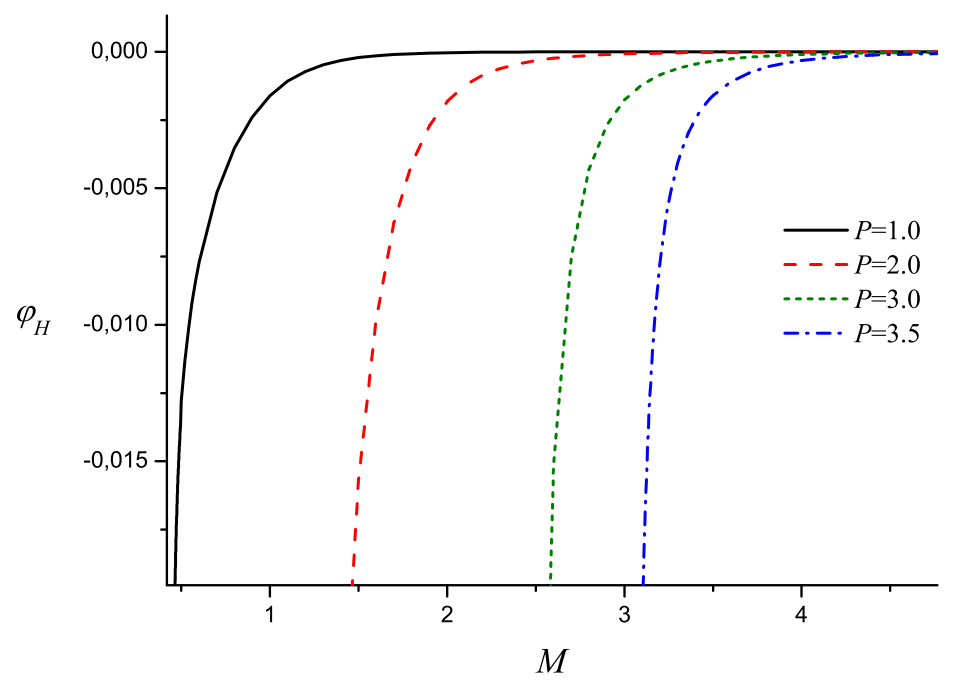

Figure 1: The value of dilaton field $\varphi$ on the horizon as a function of the mass $M$, for $P=1.0,2.0,3.0,3.5$. For low values of $M$ the absolute value of the dilaton increases considerably and prevents the formation of a degenerate horizon.

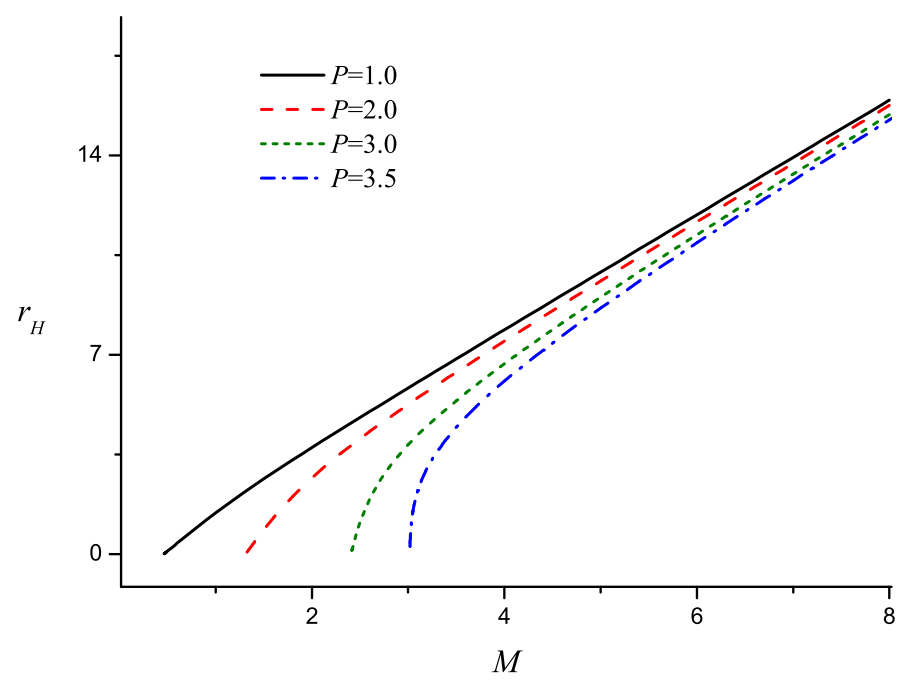

Figure 2: The $M-r_{H}$ relation for the same values of the parameters as in Fig.(11). 


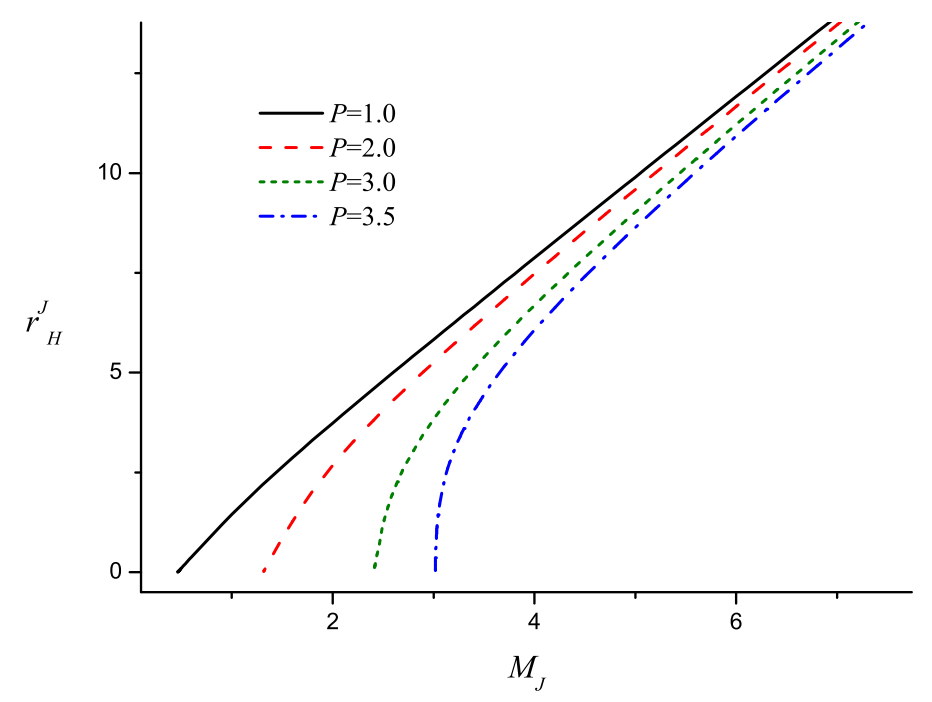

Figure 3: The $M_{J}-r_{H}^{J}$ relation, where $M_{J}$ and $r_{H}^{J}$ are respectively the mass and the radius of the black hole in the Jordan frame, for the same values of the parameters as in Fig.(11). For these values of the parameters (and $\alpha=0.01$ ) the difference from the corresponding Fig.(2) in the Einstein frame is insignificant.

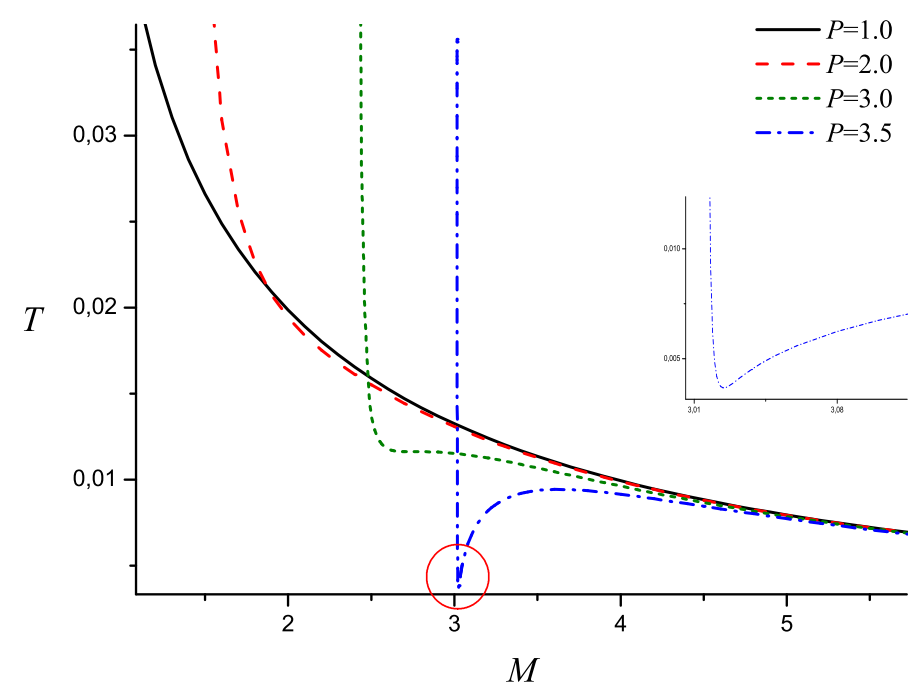

Figure 4: $M-T$ relation for the same value of the parameters as in Fig.(11). A magnification of the circled region is also shown. 